\title{
Growing Carbon Nanotubes within Boron Nitride Nanotubes
}

\author{
Raul Arenal ${ }^{1,2}$, A. Lopez-Bezanilla ${ }^{3}$ \\ 1. Laboratorio de Microscopias Avanzadas (LMA), Instituto de Nanociencia de Aragon (INA), \\ Universidad de Zaragoza, Calle Mariano Esquillor, 50018 Zaragoza, Spain \\ 2. ARAID Fundation, Calle Mariano de Luna, 50018 Zaragoza, Spain \\ 3. Argonne National Laboratory, 9700 S. Cass Av., Lemont, IL, USA
}

The controlled synthesis and growth of carbon nanotubes (CNTs) has been a long standing challenge in the fabrication of carbon based materials for electronic applications. Due to the sensitivity to small changes in their structure, the successful integration of CNTs on electronic devices is conditioned to the preservation of the graphitic network of single and multiwalled nanotubes from external perturbations [1]. It is therefore necessary to develop experimental strategies for CNT synthesis that guarantee the formation of crystalline structures of carbon materials while ensuring a protection from the environment without affecting its electronic properties. Under this context boron nitride nanotubes (BNNTs), due to their uniform electronic properties and their chemical inertness characteristics, is one of the most appropriated nanomaterials for achieving these goals. In fact BNNTs are large band gap insulators exhibiting a resistivity to oxidation up to $900{ }^{\circ} \mathrm{C}$ [2]. In the last 15 years several electron-irradiation studies have demonstrated the capability and interest of such kind of treatments for modifying and understanding the behavior of carbon-based and related nanomaterials under extreme conditions and radiation [3]. Here we report the synthesis and growth of crystalline carbon nanotubes inside a larger diameter BNNT via in-situ electron irradiation in a TEM [4].

Electron beam irradiation and high-resolution transmission electron microscopy (HRTEM) were performed using an imaging-side aberration-corrected FEI Titan-Cube microscope working at $80 \mathrm{kV}$, equipped with a Cs corrector. Complementary spatial-resolved electron energy loss spectroscopy (SREELS)-STEM studies were also carried out using a FEI Titan Low-Base microscope, working at $80 \mathrm{kV}$, which is equipped with a Cs probe corrector and ultra-bright XFEG electron source. In both cases, particular attention was devoted to avoid contamination during acquisition. Single-walled (SW) BNNT were produced by laser vaporization technique [5]. Some of these BNNT can be partially filled by amorphous carbon [5]. Furthermore, density functional theory (DFT) simulations were conducted for determining the structural stability and electronic properties of such a hybrid system.

In Figure 1, a six-frame HRTEM image sequence showing the evolution towards a nanotube structure of amorphous carbon enclosed within a BNNT. SR-EELS analyses confirm the presence of this amorphous carbon inside the NT, as displayed in Fig. 1 (g) where B-K, C-K and N-K edges are shown. In the HRTEM sequence defined by arrows, which occurs for a total cumulative dose of up to 1.8107 e-/Å2 and over a period of 380 seconds at room temperature, amorphous carbon is firstly observed in a straight BNNT and evolves over time to a crystalline structure. Simultaneously, a gradual shrinkage of the BNNT is observed. By the end of the process, the BNNT is broken and also disintegrated. We also observed the formation of an atomic-scale bridge between the tip of the carbon tube and the outer BN wall, and the subsequent reparation of the defect on both materials. Initially, a defect at the BNNT surface enhances the $\mathrm{C}$-BN interaction by establishing a connection between both tubes. The energy supplied by the electron beam favorizes the rearrangement of the external BN sidewall until complete reparation of the hexagonal network around the crystallized CNT [4]. 
In summary, these studies including HRTEM imaging, EELS analyses and DFT calculations demonstrate the feasibility of pure and crystalline carbon nanotubes growth within boron nitride nanotubes. We have investigated the formation of single-walled CNT encapsulated within single- and multi- BNNT as a pathway to construct a BNC heterostructures. The protective environment provided by the BN shell reproduce the ideal conditions for the fabrication of electronic devices in which the conducting channel remains independent of any external perturbation.

\section{References}

[1] A. Jorio, G. Dresselhaus, M.S. Dresselhaus, Springer-Verlag: Berlin, 2008.

[2] R. Arenal, X. Blase, A. Loiseau, Advances in Physics 59, 101 (2010).

[3] F. Banhart, World Scientific, Singapore (2008).

[4] A. Lopez-Bezanilla and R. Arenal, submitted.

[5] R. Arenal, O. Stephan, J.L. Cochon, and A. Loiseau, J. Am. Chem. Soc. 129, 16183 (2007).

[6] The research leading to these results has received funding from the European Union Seventh

Framework Program under Grant Agreement 312483 - ESTEEM2 (Integrated Infrastructure Initiative I3).

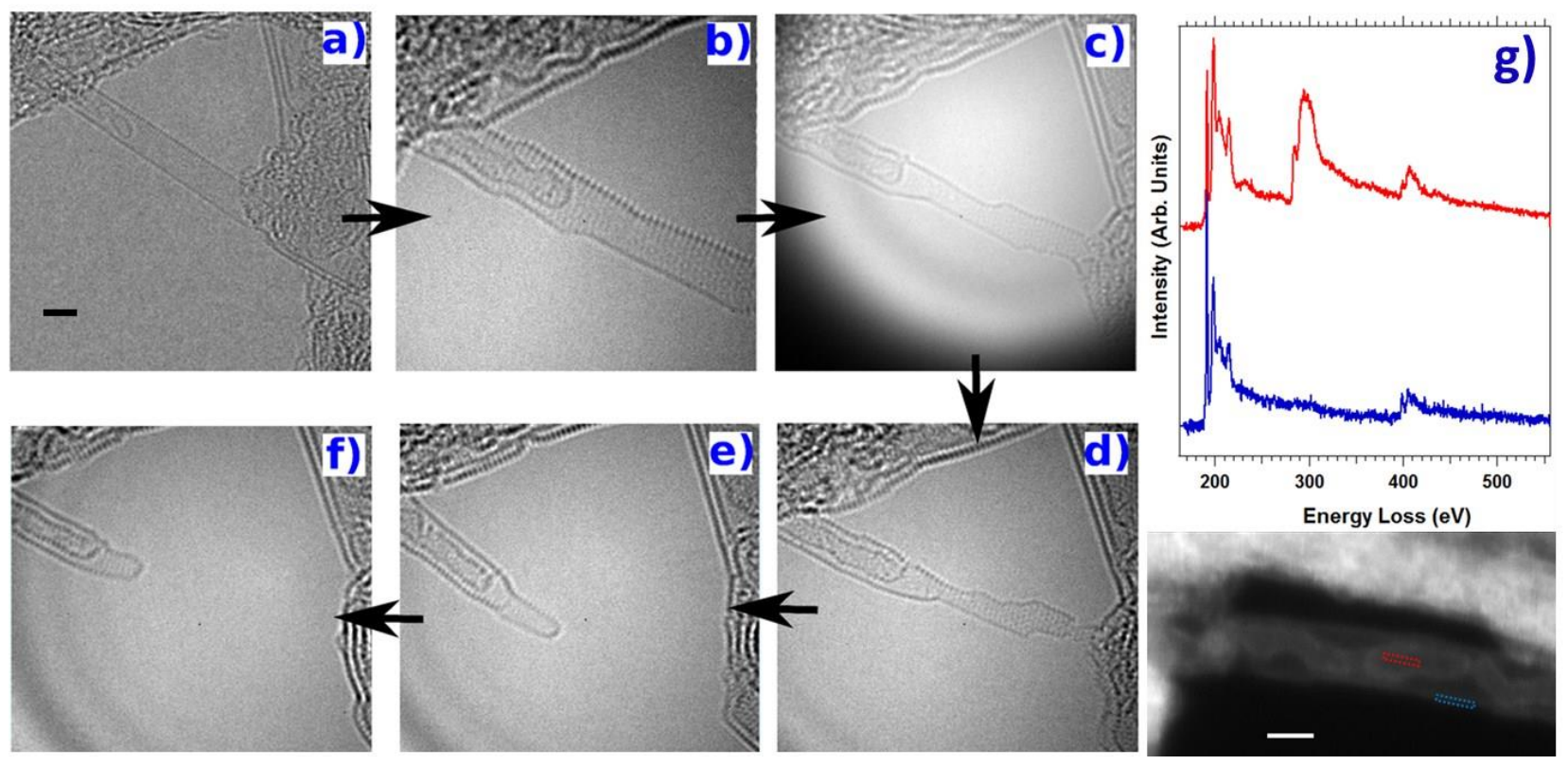

Figure 1 (a)-(f) Six-frame HRTEM image sequence displaying the formation process of a crystalline CNT from amorphous $\mathrm{C}$ encapsulated in a BNNT under electron beam irradiation (high doses for short periods of time, see Fig. 1 (c)) in a TEM. (g) EEL spectra recorded in 2 different areas (marked in the HAADF-STEM image showed below), displaying B-K, C-K and N-K edges. Scale bars in Fig. 1 (a) and (g) are $2 \mathrm{~nm}$. 